\title{
Necrosis cutánea y peneana inducidas por warfarina
}

\section{Warfarin-induced skin and penile necrosis}

\author{
Alfredo Pinzón, Catalina Arias, Ana María Cárdenas • \\ BogotÁ, D.C. (Colombia)
}

\section{Resumen}

Presentamos el caso de un hombre adulto mayor con múltiples episodios de trombosis venosa y arterial en diferentes localizaciones, quien desarrolló necrosis cutánea extensa y peneana pocos días después del uso de un antagonista de vitamina $\mathrm{K}$ (warfarina). Como parte del estudio de trombofilia se observó deficiencia de proteína C y S. (Acta Med Colomb 2012; 37: 142-146)

Palabras clave: warfarina, trombosis venosa y arterial, necrosis cutánea y peneana.

\begin{abstract} deficiency. (Acta Med Colomb 2012; 37: 142-146)

Los agentes anticoagulantes orales han sido utilizados ampliamente en la prevención primaria o secundaria de múltiples entidades tromboembólicas. Sin embargo, las consecuencias adversas de su uso pueden llegar a ser devastadoras, como sucede con los eventos trombóticos paradójicos inducidos por estos fármacos, que aumentan la morbilidad y en algunos casos la mortalidad de los pacientes que los padecen. Los eventos adversos asociados con el uso de anticoagulantes orales, particularmente la warfarina, incluyen sangrado, priapismo, hepatitis, erupción maculopapular pruriginosa, alopecia y necrosis cutánea.
\end{abstract}

We present the case of an elderly man with multiple episodes of venous and arterial thrombosis in different locations, who developed extensive skin and penile necrosis few days after the use of a vitamin $\mathrm{K}$ antagonist (warfarin). As part of the thrombophilia study, we observed protein $\mathrm{C}$ and $\mathrm{S}$

Keywords: warfarin, venous and arterial thrombosis, skin and penile necrosis.
Dr. Alfredo Pinzón Junca: Especialista en Medicina Interna, Hospital Universitario de La Samaritana; Profesor Clínico Pontificia Universidad Javeriana y Universidad de La Sabana; Dra. Catalina Arias: Residente II de Medicina Interna, Hospital Universitario de La Samaritana, Universidad de La Sabana; Dra. Ana María Cárdenas: Residente III de Medicina Interna, Hospital Universitario de La Samaritana, Universidad de La Sabana. Bogotá, D.C. (Colombia).

Correspondencia. Dr. Alfredo Pinzón, Bogotá, D.C. (Colombia).

E-mail: alfredo.pinzon@hus.org.co Recibido: 30/X/2011 Aceptado: 24/VIII/2012

\section{Descripción del caso}

Hombre de 64 años, agricultor, que presentó cuadro de dolor precordial tipo ardor, opresivo intermitente, de intensidad 6/10, no irradiado, asociado a disnea y deterioro de la clase funcional a II/IV, por lo que fue remitido al hospital de segundo nivel donde sospecharon neumonía adquirida en la comunidad por la presencia de opacidades alveolares en la radiografía de tórax y leucocitosis de $18.000 / \mathrm{mm}^{3}$ (con neutrofilia de $81 \%$ ), para lo que iniciaron tratamiento con ampicilina sulbactam; allí tomaron electrocardiograma inicial que reportaron sin signos de isquemia miocárdica. Adicionalmente presentó dolor y edema en piernas con signos clínicos de trombosis venosa, la cual fue confirmada por ultrasonido dúplex venoso que informó trombosis venosa profunda aguda infrapatelar bilateral, por lo que sospecharon que el cuadro torácico correspondía a tromboembolismo pulmonar, y decidieron iniciar anticoagulación con heparina de bajo peso molecular y warfarina. Al día siguiente presentó cambios en el electrocardiograma dados por inversión de la onda $\mathrm{T}$ en pared inferior y anterior, interpretados como isquemia miocárdica anteroseptal e inferior. Un día más tarde (es decir, dos días después del inicio de la warfarina) el paciente presentó cuadro agudo de priapismo, asociado a dolor, cianosis y edema del glande, por lo que suspendieron el tratamiento con warfarina y nitratos, y remitieron para manejo integral en tercer nivel, cuando habían transcurrido seis días desde el inicio del cuadro clínico.

$\mathrm{Al}$ ingreso el paciente se encontraba con signos vitales dentro de límites normales, hemodinámicamente estable, con leucocitosis de $21230 / \mathrm{mm}^{3}$ (neutrofilia de $89 \%$ sin formas inmaduras, ni alteración de otras líneas celulares) sin evidencia de otros signos de respuesta inflamatoria sistémica. Se evidenciaba necrosis del glande hasta el surco balanoprepucial, con esfacelación de piel superficial, cuerpos cavernosos indurados, eritema en bolsa escrotal, y edema de pene y escroto (Figura 1). Al tacto rectal se palpó una próstata grado I de aproximadamente $20 \mathrm{~g}$. Se encontró edema grado II de miembros inferiores con signos clínicos 


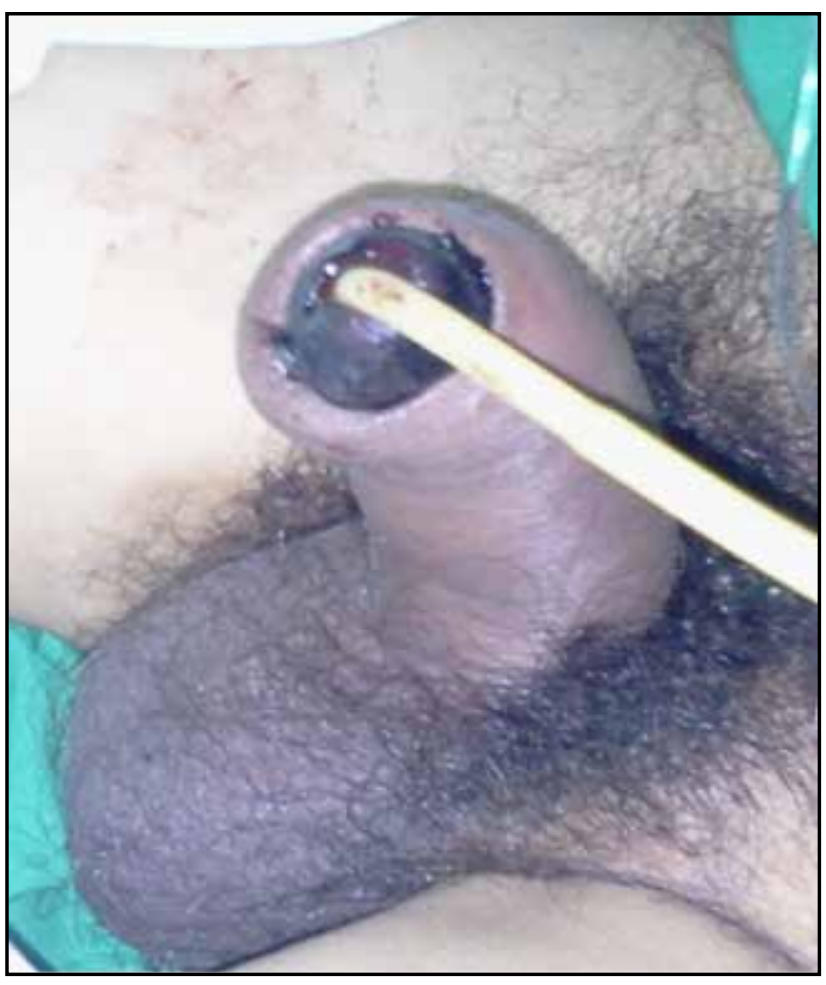

Figura 1. Necrosis del glande.

de trombosis venosa profunda. Fue manejado inicialmente con adrenalina intracavernosa y lavados de cuerpos cavernosos. En ese momento no había otros hallazgos positivos al examen físico.

A la revisión por sistemas refería síntomas urinarios obstructivos bajos dados por micción prolongada y goteo posmiccional ocasional.

Entre los antecedentes patológicos, el paciente tenía hipertensión arterial y enfermedad pulmonar obstructiva crónica, así como exposición al humo de leña por 30 años; se encontraba en manejo con enalapril $20 \mathrm{mg}$ qd y verapamilo $120 \mathrm{mg}$ qd. No refería algún otro antecedente personal o familiar.

Entre los paraclínicos iniciales se encuentran:

- Hemograma: Hto: 36.7\%; Hb: 12.3 g/dL; leucocitos: $21230 / \mathrm{mm}^{3}$ (neutrófilos $89 \%$ y linfocitos $6 \%$ ); plaquetas: $246000 / \mathrm{mm}^{3}$.

- Química sanguínea: nitrógeno ureico: $13.02 \mathrm{mg} / \mathrm{dL}$; creatinina sérica: $0.46 \mathrm{mg} / \mathrm{dL}$; glucemia: $71 \mathrm{mg} / \mathrm{dL}$; natremia: $137 \mathrm{mEq} / \mathrm{L}$; caliemia: $4.3 \mathrm{mEq} / \mathrm{L}$.

- Uroanálisis: leucocitos: $>100 x c$; bacterias: +++; nitritos: positivo.

- VDRL: no reactiva.

- Tiempos de coagulación: TP: 17.7 s (control 12 s); INR: 1.39; TPT: $42 \mathrm{~s}$ (control $28 \mathrm{~s}$ ).

- Troponina I: negativa

- Fibrinógeno: $353 \mathrm{mg} / \mathrm{dL}$.

- Anticuerpos antinucleares: negativos.

- Anticoagulante lúpico: 35.1 s.
- Anticardiolipinas: IgG: 22.70 GLP (control a las 12 semanas: 9.30 GLP); IgM: 11.90 GLP (control a las 12 semanas: 6.70 GLP) [valor negativo < 20 GLP].

- Antitrombina III: $31.1 \mathrm{mg} / \mathrm{dL}$ [valor de referencia: 16.6-31.4].

- $\quad \beta-2$ microglobulina: $2.9 \mathrm{mg} / \mathrm{L}$ [valor de referencia: 1.0-3.0].

- Coombs directo: negativo.

- Factor reumatoide: negativo.

- Crioglobulinas: negativo.

Por la sospecha de tromboembolismo pulmonar se realizó angiotac de tórax el cual fue negativo para defectos de llenamiento que soportaran ese diagnóstico; informaron un patrón en vidrio esmerilado confluente en algunos segmentos pulmonares semejando un patrón de empedrado que ocasionaba disminución del parénquima pulmonar en los segmentos posterior del lóbulo superior derecho, lóbulo medio y segmento basal anterior del lóbulo inferior izquierdo, más discreto en el segmento basal lateral y basal posterior del lóbulo inferior derecho, lo que parecía corresponder a un proceso de alveolitis activa y ocupación del espacio aéreo pulmonar.

Tres días después del ingreso al hospital de III nivel, el paciente presentó flictena violácea que cubría todo el aspecto posterior de la pierna izquierda (Figura 2). Por esto, y teniendo en cuenta todo el cuadro clínico, se consideró que podía estar cursando con un estado protrombótico, por lo que se complementaron los estudios de hipercoagulabilidad (las muestras fueron obtenidas seis días después del inicio de la warfarina y tras cuatro días de su suspensión), entre los cuales debía descartarse el síndrome de Trousseau asociado a neoplasia oculta, teniendo en cuenta la edad del paciente. Se obtuvieron los siguientes resultados:

- Hemocultivo: Staphylococcus epidermidis meticilino resistente.

- Proteína C: $67.5 \%$ (valor de referencia: 70-140\%).

- Proteína S: 47.9\% (valor de referencia: 75-130\%).

Durante su evolución el paciente presentó progresión de las lesiones violáceas hacia la necrosis (Figura 3), además de presentar posterior compromiso de la pierna derecha con

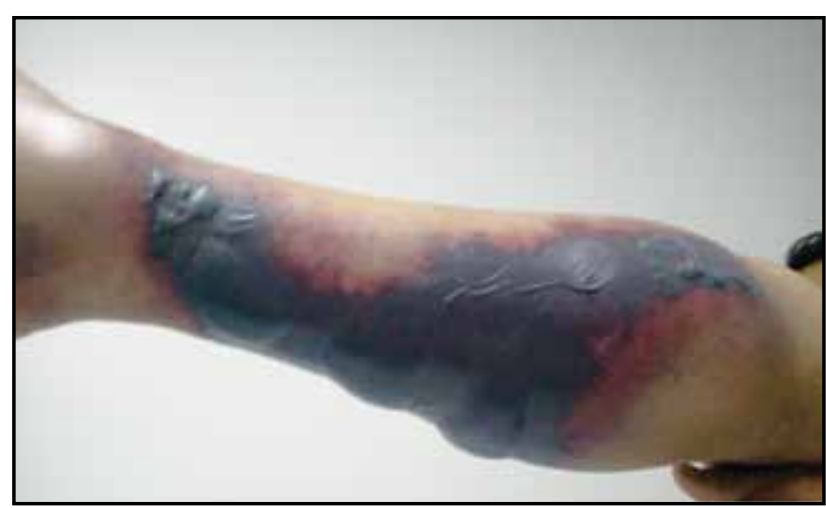

Figura 2. Bula hemorrágica bien delimitada en pierna izquierda 
equimosis extensa en su cara lateral y posterior del tercio medio de ésta, así como la presencia de múltiples flictenas hemorrágicas que fueron drenando espontáneamente.

Así mismo, presentó nuevas lesiones con signos de infección en dorso, muslos y glúteos, para lo que se inició tratamiento con meropenem y vancomicina. Se consideró que la aparición de todas las lesiones necróticas posterior a la administración de warfarina podría estar en relación con deficiencia de proteína $\mathrm{C}$ de la coagulación.

Posteriormente presentó deterioro de su estado general que lo llevó a requerir manejo en la unidad de cuidados intensivos donde cursó con extensión de las lesiones de los miembros inferiores llegando hasta la necrosis. Se tomó biopsia de la lesión, la cual evidenció necrosis epidérmica focal (Figura 4), hemorragia dérmica difusa y trombosis de venas pequeñas (Figura 5).

Dentro de los estudios adicionales se realizó una endoscopia de vías digestivas altas y una tomografía abdominal, las cuales descartaron la presencia de neoplasias; el antígeno prostático específico se encontró en el rango normal.

Doce días después de haber recibido la warfarina aparecieron lesiones de las mismas características en la región dorsolumbar y caderas, las cuales, igualmente, progresaron a la necrosis cutánea (Figura 6). Dado el importante compromiso de la superficie corporal, presentó infección bacteriana con bacteriemia por Enterobacter cloacae.

Se realizó inicialmente manejo con desbridamiento enzimático de las áreas necróticas con nitrofurazona, y posteriormente fue llevado a desbridamiento quirúrgico donde se evidenció necrosis del músculo peronero largo, por lo que se consideró cuadro de miositis para lo cual recibió nuevamente tratamiento antibiótico de amplio espectro.

Por la necrosis del glande fue llevado a penectomía parcial con glandectomía, y tres días más tarde se realizó amputación supracondílea por amplio defecto de cobertura de aproximadamente $20 \times 15 \mathrm{~cm}$ en dorso de pierna izquierda, con exposición de tejido muscular y vientre muscular del gastrocnemio necrótico, con exposición tendinosa necrótica.

Después del posoperatorio hubo una adecuada evolución clínica, por lo que egresó para continuar tratamiento ambulatorio.

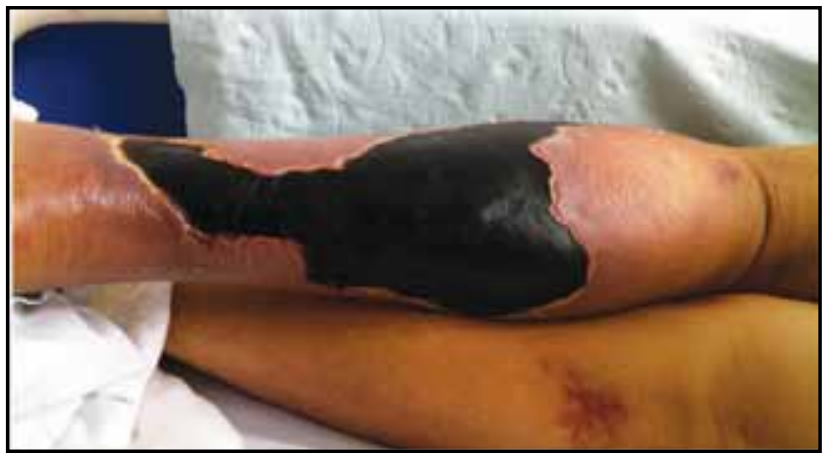

Figura 3. Úlcera necrótica de pierna izquierda

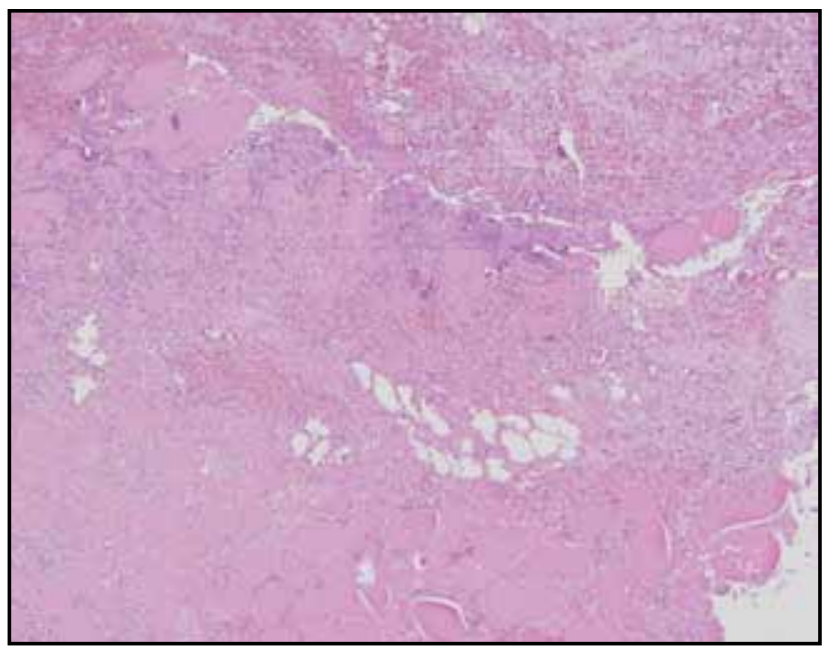

Figura 4. Necrosis epidérmica focal (Tinción Hematoxilina-Eosina, aumento 10X)

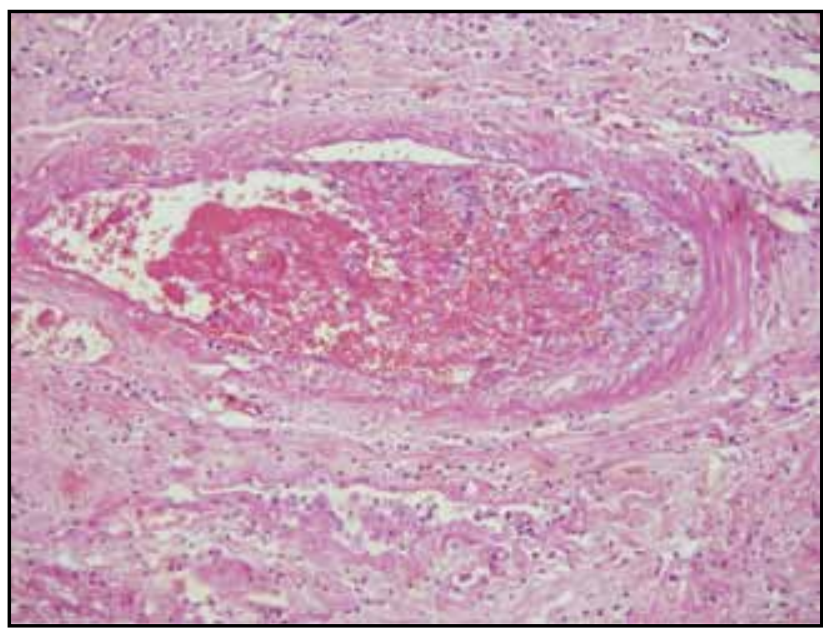

Figura 5. Trombosis de pequeños vasos con recanalización (Tinción Tricromo, aumento 20X)

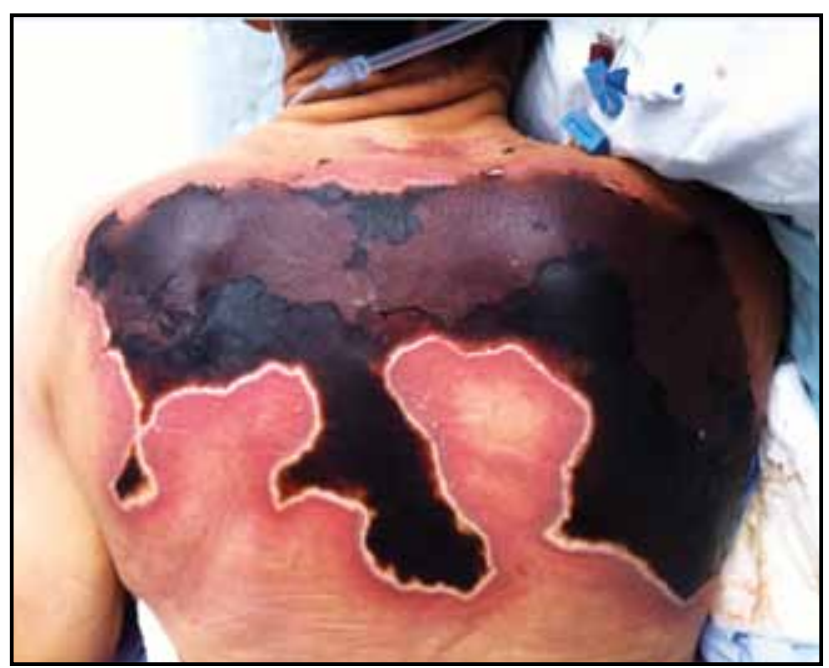

Figura 6. Escara necrótica en dorso 


\section{Discusión}

Los anticoagulantes orales que actúan antagonizando la vitamina $\mathrm{K}$ se han utilizado durante mucho tiempo en la práctica clínica. Sin embargo, las dificultades relacionadas con su manejo y sus serias complicaciones aún persisten. Entre estas últimas, el sangrado es ampliamente conocido, pero también deben tenerse en cuenta otras, tal como la necrosis cutánea inducida por warfarina, dada su alta morbilidad y mortalidad.

La necrosis cutánea es un evento adverso raro pero serio de la anticoagulación con antagonistas de la vitamina K, como el acenocumarol o la warfarina. Se comunicó por primera vez en 1916 en el trabajo de McLean y posteriormente en 1943 por Flood et al, denominándose "tromboflebitis migrans diseminada" de la mama. Su incidencia varía entre 0,01 y $0,1 \%$ en los pacientes tratados con estos medicamentos; habiéndose reportado aproximadamente 300 casos en los últimos 30 años a nivel mundial. Esta complicación tiene alta propensión a comprometer áreas corporales con incremento en la grasa subcutánea, como el abdomen, los glúteos, muslos, piernas y tejido mamario en las niñas y mujeres afectadas. Sin embargo, también se han reportado presentaciones en el pene, los brazos, y el dorso. Aproximadamente un tercio de los pacientes tienen compromiso de múltiples regiones corporales. Demográficamente, las mujeres que se encuentran en tratamiento para embolia pulmonar o tromboflebitis son las más afectadas por este efecto adverso del tratamiento anticoagulante (1-3).

La fisiopatología de la necrosis cutánea inducida por warfarina es aún desconocida y sus causas todavía no han sido completamente definidas. Sin embargo, el mecanismo más frecuentemente involucrado es la presencia de un estado hipercoagulable paradójico a través de la inhibición de algunos factores vitamina $\mathrm{K}$ dependientes mediada por la warfarina, lo que lleva a un desbalance entre factores anti y procoagulantes; el descenso más rápido de la proteína $\mathrm{C}$ y del factor VII se explica por sus vidas medias más cortas, de 5 a 8 horas, con respecto a la de otros factores como el II, IX y X, cuyas vidas medias varían de 24 a 72 horas. La deficiencia de proteína C, proteína $\mathrm{S}$ y factor VII representan un factor de riesgo para su presentación; también se han planteado reacciones de hipersensibilidad a la warfarina, aunque las pruebas cutáneas han producido hallazgos negativos en estos pacientes (4).

La presentación clínica tiene un curso agudo con parestesias y edema, seguido de petequias y equimosis, que progresan a una bula hemorrágica bien delimitada en un término de 24 horas. Finalmente, se presenta necrosis de coagulación de todo el grosor de la piel, con formación de úlceras subcutáneas profundas en los cinco días siguientes al inicio de la terapia anticoagulante. Sin embargo, existen reportes de caso en los que las complicaciones se han presentado 10 y hasta 15 días después del inicio de la terapia o incluso varios días después de su suspensión. Los hallazgos histopatológicos más importantes comprenden la presencia de microtrombos difusos en los capilares dérmicos y subcutáneos, vénulas y venas profundas, con daño de las células endoteliales, resultando en una necrosis cutánea isquémica y una extravasación importante de glóbulos rojos $(2,5)$.

Las lesiones de la necrosis cutánea inducida por warfarina pueden ser difíciles de diferenciar de otras, pero la biopsia de piel y una cuidadosa consideración de la historia clínica, incluyendo el tiempo de aparición (relación de temporalidad con la administración del anticoagulante), distribución dérmica de las lesiones y hallazgos de laboratorio, son esenciales para el diagnóstico oportuno y tratamiento adecuado del paciente. El reconocimiento temprano de esta afección puede salvar una extremidad, permitiendo el inicio temprano de medidas terapéuticas como la descontinuación inmediata del medicamento, que mejora el pronóstico pues detiene la progresión de la necrosis. La base principal del tratamiento es el uso de plasma fresco y vitamina K para revertir el efecto de la warfarina y restaurar los niveles de proteína $\mathrm{C}$ y $\mathrm{S}$, así como el uso de un anticoagulante alterno como la heparina. El tratamiento local incluye el uso de antibacterianos tópicos, aunque el desbridamiento quirúrgico, con o sin injertos cutáneos, o incluso las amputaciones se han requerido en más de $50 \%$ de los casos. Se ha descrito el uso de concentrados de anticuerpos monoclonales purificados de proteína $\mathrm{C}$ en pacientes con deficiencia de la misma; sin embargo, su alto costo lo ha hecho de uso restringido (2).

Como se mencionó, al iniciar la terapia con warfarina puede haber un efecto procoagulante debido a una inhibición más rápida de la proteína $C$. Se ha sugerido que en pacientes con trombocitopenia inducida por heparina (TIH) la warfarina puede precipitar gangrena venosa de las extremidades y/o necrosis cutánea inducida por este fármaco. Srinivasan y colaboradores describieron seis pacientes con TIH en quienes se presentaron estos dos eventos. Al iniciar la warfarina, cinco tenían TIH y en uno ésta se reconoció más adelante. Las complicaciones aparecieron después de dos a siete días, y consistieron en necrosis cutánea inducida por warfarina $(n=5)$ y gangrena venosa de piernas $(n=2)$; un paciente tenía las dos. Todos los pacientes tenían INR en rango terapéutico. Una paciente requirió amputación de pierna y mama, y otro murió. Dado que la warfarina puede precipitar gangrena venosa y/o necrosis cutánea en el estado hipercoagulable de la TIH, se debe evitar iniciarla sin oposición (otro anticoagulante simultáneo) y debe iniciarse a dosis bajas después de la recuperación de la trombocitopenia. Estas implicaciones se pueden extender a otras trombofilias (6).

Talbert y colaboradores reportaron el caso de un hombre joven con trombosis venosa profunda bilateral e isquemia peneana recurrente que requirió penectomía distal e injertos de piel; después de la cirugía el paciente se cambió de enoxaparina a warfarina y nueve días más tarde desarrolló úlceras necróticas en brazos, que posteriormente mostraron ser secundarias a necrosis cutánea inducida por warfarina. A pesar de la alta sospecha, no se demostró serológicamente ningún trastorno de hipercoagulabilidad (7). 
En Corea, el grupo de Chang describió el caso de un hombre de 56 años con adenocarcinoma de pulmón y embolismo pulmonar cuyo recuento inicial de plaquetas era de 531000/L. El paciente fue anticoagulado con heparina de bajo peso molecular (HBPM) y al día siguiente se inició warfarina (5 mg qd), descontinuando la HBPM al cuarto día. Al tercer día de iniciada la anticoagulación oral desarrolló edema en pierna izquierda y priapismo doloroso de 24 horas de duración; su recuento de plaquetas alcanzó un nadir de 164000/L. El paciente tenía deficiencia de proteínas C y S, con nivel de actividad de $16-20 \%$, respectivamente. Se suspendió la warfarina y se realizó drenaje de cuerpos cavernosos, con lo que mejoró el priapismo; sin embargo, al día siguiente empezó necrosis del pene y el glande, que posteriormente requirió penectomía parcial (8).

Nuestro caso reportado muestra un adulto mayor hipertenso y con neumopatía crónica, quien presentó un cuadro infeccioso pulmonar y en forma concomitante trombosis venosa profunda de miembros inferiores (sin factores protrombóticos previos identificados), lo que indicó el uso de anticoagulación parenteral y oral, tras lo cual presentó dos infrecuentes reacciones adversas relacionadas con el uso de la warfarina: necrosis cutánea severa que llevó a sepsis de tejidos blandos, y priapismo refractario que ocasionó necrosis del glande, ambas con considerable morbilidad y discapacidad, pero con resultado final satisfactorio considerando la supervivencia del paciente. El diagnóstico se basó en el cuadro clínico característico, la demostrada relación de temporalidad entre la administración del fármaco y la aparición de los síntomas, y los hallazgos histológicos en la biopsia de piel.

Se descartaron otros diagnósticos diferenciales, como la TIH (ya que nunca presentó trombocitopenia absoluta ni relativa), síndrome antifosfolípido (anticardiolipinas y anticoagulante lúpico negativos) y síndrome de hipercoagulabilidad secundario a neoplasias sólidas o hematológicas (estudios complementarios y de seguimiento hematológico negativos). Se excluyó el efecto protrombótico paradójico inicial de la warfarina como causa de la trombosis venosa profunda ya que ésta se documentó antes del inicio del antagonista de la vitamina $\mathrm{K}$.

Aunque en este caso se observó disminución de las proteínas $\mathrm{C}$ y $\mathrm{S}$, aún no se puede afirmar categóricamente que la deficiencia de esas proteínas de la coagulación haya sido la única causa precipitante del cuadro, ya que cabría esperar un nivel de actividad de la proteína $\mathrm{C}$ más bajo, y además dichos niveles se midieron durante el episodio de trombosis venosa, así como poco tiempo después del uso de warfarina, cuando aún podría haber actividad residual de este fármaco. Es necesario repetir estas mediciones posteriormente para afianzar el diagnóstico de esta trombofilia.

Este caso nos recuerda la imperiosa necesidad de estar atentos a la posibilidad de complicaciones infrecuentes pero catastróficas y potencialmente fatales asociadas a un medicamento de uso común, como la warfarina.

\section{Conclusiones}

La necrosis inducida por warfarina es una reacción adversa rara pero severa secundaria al tratamiento con antagonistas de la vitamina $\mathrm{K}$.

Debe ser reconocida y tratada a tiempo para evitar las consecuencias devastadoras de este efecto adverso de los anticoagulantes orales cumarínicos.

$\mathrm{La}$ isquemia y necrosis del pene, secundaria a necrosis cutánea inducida por warfarina, es una reacción adversa aún más infrecuente, y devastadora.

Aunque se sospecha con frecuencia, no siempre es posible demostrar una trombofilia asociada a la necrosis cutánea inducida por warfarina.

\section{Agradecimientos}

Al Departamento de Patología del Hospital Universitario de La Samaritana, por su colaboración con los estudios histológicos.

\section{Referencias}

1. Reyes A, Raffo M, Masia G, et al. Necrosis cutánea por tratamiento anticoagulante oral: caso clínico. Rev Chil Cir 2011; 63(2): 200-203.

2. Nazarian RM, Van Cott EM, Zembowicz A, et al. Warfarin-Induced Skin Necrosis. J Am Acad Dermatol 2009; (61)2: 325-332.

3. Horn JR, Danziger LH, Davis RJ. Warfarin-induced skin necrosis: report of four cases. Am J Hosp Pharm 1981; 38(11): 1763-8.

4. Da Silva JM, Thomazhino F, Sardinha WE, et al. Necrose cutânea induzida por antagonistas da Vitamina K. J Vasc Bras 2009; 8(4): 343-348.

5. Wynn SS, Jin DK, Essex DW. Warfarin-induced skin necrosis occurring four days after discontinuation of warfarin. Haemostasis 1997; 27(5): 246-50.

6. Srinivasan AF, Rice L, Bartholomew JR, et al. Warfarin-Induced Skin Necrosis and Venous Limb Gangrene in the Setting of Heparin-Induced Thrombocytopenia. Arch Intern Med 2004; 164: 66-70.

7. Talbert M, Wood H. Penile Ischemia and Loss Due to Warfarin-Induced Skin Necrosis. Urology 2011; 77(2): 335-336.

8. Chang IH, Soo-Ha M, Hoon-Chi B, et al. Warfarin-Induced Penile Necrosis in a Patient with Heparin-Induced Thrombocytopenia. J Korean Med Sci 2010; 25: 1390-1393. 